\title{
Efficacy of a Tailored Moisturizer for Reducing Chemotherapy-Induced Skin Dryness in Breast Cancer Patients: A Randomized Controlled Trial
}

\section{Danbee Kang}

Sungkyunkwan University

Nayeon Kim

Samsung Medical Center

Young Hyuck Im

Samsung Medical Center

Yeon Hee Park

Samsung Medical Center

Ji Yeon Kim

Samsung Medical Center

Hyeokgon Park

Amorepacific (South Korea)

Eunjoo Kim

Amorepacific (South Korea)

Di Zhao

Johns Hopkins University

Eliseo Guallar

Johns Hopkins University

Jin Seok Ahn

Samsung Medical Center

Juhee Cho ( $\nabla$ jcho@skku.edu )

Sungkyunkwan University

\section{Research Article}

Keywords: Breast neoplasm, chemotherapy, xeroderma, emollients, randomized controlled clinical trial

Posted Date: March 8th, 2021

DOI: https://doi.org/10.21203/rs.3.rs-150834/v1 
License: (c) (1) This work is licensed under a Creative Commons Attribution 4.0 International License. Read Full License

Version of Record: A version of this preprint was published at Journal of the American Academy of Dermatology on October 1st, 2021. See the published version at https://doi.org/10.1016/j.jaad.2021.10.047. 


\section{Abstract}

Introduction: While up to $60 \%$ of breast cancer patients undergoing chemotherapy experience skin side effects reducing quality of life, it was unclear which individual product was beneficial. To evaluate the effectiveness of a tailored moisturizer with a high content (1.5\%) of pseudo-ceramides on skin dryness due to chemotherapy in breast cancer patients.

Methods: Randomized controlled trial conducted from February 2015 to October 2018. 204 breast cancer patients experiencing skin dryness after 1 cycle of chemotherapy were randomly assigned to 3 groups (tailored moisturizer with high pseudo-ceramide content; general moisturizer; and usual care). Skin dryness, dullness, quality of life, and changes in sebum level were assessed at baseline, 3 weeks, and 1 month after completion of chemotherapy. The primary objective of the trial was to compare the tailored moisturizer group to usual care.

Results: At 1 month after completion of chemotherapy, the tailored moisturizer group was significantly less likely to report severe skin dryness compared to the usual care group ( 8.5 vs. $27.9 \%$, respectively, $P<$ 0.01). The tailored moisturizer group reported significantly lower levels of skin dullness and lower levels of impairment of dermatological quality of life compared with the other two groups.

Conclusions: A tailored moisturizer with a high content of pseudo-ceramides improved skin dryness, dullness and dermatological quality of life compared to usual care in breast cancer patients undergoing chemotherapy.

\section{Introduction}

The majority of breast cancer patients receive standard chemotherapy regimens including doxorubicin, cyclophosphamide, fluorouracil, or taxotere (docetaxel) ${ }^{1,2}$. These regimens target rapidly dividing cells ${ }^{3}$ and produce a variety of cutaneous adverse effects. The most common skin symptoms reported by breast cancer patients treated with chemotherapy are skin dryness (57.9\%) and dullness $(49.1 \%){ }^{4}$, but these patients also report alopecia, erythema, pruritus, macular eruptions, hyperpigmentation, and desquamation ${ }^{5-11}$. The cutaneous adverse effects of cancer treatment are not only very common, but also often visible on the face, arms, and/or upper torso, impairing body image and quality of life of breast cancer patients. Moreover, these adverse effects can be directly responsible for treatment discontinuation, reduced compliance and therapeutic success rates. ${ }^{12}$

A board of experts recommended the use of clinically tested skin care interventions to manage cutaneous reactions prior to, during, and after cancer therapy ${ }^{13}$. However, the evidence on interventions such as urea-based cream ${ }^{14}$ or fluorouracil $5 \%$ cream ${ }^{15}$ is limited by the studies that lacked a control group, by the focus on targeted therapy, or by the simultaneous use of multiple products ${ }^{16,17}$, so that it was unclear which individual product was beneficial. Moreover, there is no randomized evidence evaluating the 
efficacy of any tailored intervention for managing specific skin problems such as chemotherapy-induced dryness.

A recent cohort study of breast cancer patients undergoing chemotherapy found that skin dryness after chemotherapy was significantly associated with decreased skin sebum content ${ }^{4}$. Chemotherapy drugs may decrease sebum production from sebaceous glands and impair the skin barrier function with unpleasant symptoms. Furthermore, the disrupted barrier function and water deficiency that occurs in the skin of patients undergoing chemotherapy can be repaired by the topical application of natural ceramides 18 or synthetic pseudo-ceramides ${ }^{19}$.

We thus designed a randomized controlled trial to test the efficacy of a tailored moisturizer with a high content $(1.5 \%)$ of pseudo-ceramides for managing chemotherapy-induced dryness compared to usual care in patients with breast cancer receiving adjuvant chemotherapy ${ }^{19}$. As a secondary comparison, we compared the tailored moisturizer with a general moisturizer.

\section{Results}

Among 791 patients initially eligible, 502 patients (63\%) agreed to participate in the trial. After excluding 254 patients (51\%), we randomized 247 participants ( 82 to the tailored moisturizer, 82 to usual care, and 83 to the general moisturizer) (Figure 1). Forty three patients withdrew prior to the baseline visit, leaving 204 patients in the study. After randomization, 16 patients withdraw because of inconvenience related to participating in the study, and 12 patients were lost to follow-up for the visit at 1 month after completion of chemotherapy, leaving 176 participants in the trial (tailored, $N=59$; usual care, $N=61$; general, $N=56$ ).

The average age of study participants was 48.2 years, and $85.2 \%$ of participants had stage I or II breast cancer. Patient characteristics were well balanced across study groups (Table 1). The proportion of patients with severe skin dryness at baseline in the tailored, usual care, and general moisturizer groups were 26.7, 21.9, and 35.4\%, respectively (Figure 2). At 1 month after completion of chemotherapy, the tailored moisturizer group was significantly less likely to report severe skin dryness compared to the usual care group ( 8.5 vs. $27.9 \%$, respectively, $P<0.01)$, but similar to the general moisturizer group $(8.9 \%, P=$ 0.93) (Figure 2).

Compared to the tailored moisturizer group, sebum content in the usual care group was significantly lower at 3 weeks and at 1 month after completion of chemotherapy (Table 2 and Figure 3A). The general moisturizer group also showed lower sebum content compared with the tailored moisturizer group at 3 weeks and at 1 month after completion of chemotherapy, but the difference at 1 month after completion of chemotherapy was not statistically significant (Table 2 and Figure 3B). The change in water content showed a similar pattern to the change in sebum content, but none of the differences across the 3 groups were statistically significant (Table 2).

At 1 month after completion of chemotherapy, patients in the tailored moisturizer group reported significantly lower levels of skin dryness (mean score 3.8) than those in the usual care group (mean score 
5.4, $P=0.001$ ), but similar to those in the general moisturizer group (mean score 4.4, $P=0.18$ ) (Table 3 ). The tailored moisturizer group also reported significantly lower levels of skin dullness (mean score 3.1) than both comparison groups (usual care group 4.6, $P=0.012$; general moisturizer group 4.4, $P=0.024$ ). The tailored moisturizer group also reported significantly lower levels of impairment of dermatological quality of life (mean score 2.0) than both comparison groups (usual care group 4.8, $P<0.001$, general moisturizer group $3.9, P=0.018$ ).

In the tailored and general moisturizer groups, 13 (22.0\%) and 11 (19.6\%) patients skipped moisturizer use for three consecutive days or more, respectively. In the usual care group, the median number of cosmetic products per day by patients in the usual care group was 6 (Supplement Table 1).

There were no participants who had unintended effects in this study.

\section{Discussion}

In this randomized controlled trial, breast cancer patients undergoing chemotherapy assigned to a tailored moisturizer with a $1.5 \%$ pseudo-ceramide content to prevent loss of sebum levels reported less dryness, less dullness, less stress, and better dermatological quality of life at 1 month after completion of chemotherapy compared to patients assigned to usual care. In addition, the usual care group experienced a significant reduction in sebum content compared to the tailored moisturizer group. Patients in the tailored moisturizer group also experienced significantly less dullness and better dermatological quality of life at 1 month after completion of chemotherapy compared to the general moisturizer group.

Chemotherapy induces skin changes comparable to accelerated aging, including increased skin fragility 20. Chemotherapy drugs decrease sebum production in the sebaceous glands and cause loss of the skin barrier function. This results in loss of moisture and lipids in the stratum corneum of the epidermis with a sensation of skin dryness. General moisturizers and other cosmetics which provide only lipid or water content may temporarily increase the oil and water content in the stratum corneum of the epidermis, but this is insufficient to maintain effective skin sebum levels and to improve the barrier function of the skin deteriorated by chemotherapy.

We hypothesized that a tailored moisturizer with a high level of pseudo-ceramides would prevent loss of moisture and lipids by improving skin the barrier function of the epidermis ${ }^{21,22}$. The tailored pseudoceramide moisturizer in our study closely mimicked the composition of skin lipids, which include fatty acids, triglycerides, and cholesterol, which may also contribute to more effective absorption and to a synergistic effect of lipids and moisture. Indeed, the usual care and the general moisturizer groups in our study had a more marked decrease in sebum content compared to the tailored moisturizer group, although only the difference between the usual care and the tailored moisturizing group was statistically significant.

Restoration of skin ceramide levels is also under development to manage other skin side effects of chemotherapy in addition to dryness ${ }^{23,24}$. In our study, patients in the tailored moisturizer group reported 
less distress due to skin problems and better dermatological quality of life during chemotherapy compared with other groups. While the efficacy of ceramides in improving skin function has been shown in many animal and clinical studies ${ }^{19,23,25}$, there was little evidence in chemotherapy-induced skin changes. Additional studies with larger sample sizes are needed to evaluate the effect of ceramides in different cancer patients and to better understand the molecular mechanisms underlying lipid barrier restoration in chemotherapy induced skin changes.

In our study, patients in the usual care group used an average of 6 cosmetics to prevent potential skin problems during chemotherapy, but they still experienced more dryness and other skin problems compared to the other study groups. It is possible that the products that they used had low skin absorption, lacked an adequate lipid content, or aimed only at immediate correction of skin dryness. These products may improve skin dryness temporarily but fail to restore skin lipids and the skin barrier function in breast cancer patients with chemotherapy. In addition, patients in the usual care may have used cosmetics on demand, while about $80 \%$ of the tailored moisturizer group used the intervention consistently throughout the study period.

There are several limitations to our study. Patients who had more sensitive skin or who were more interested in skin health may have been more likely to participate in our study. Furthermore, study participants may be more likely to report skin-related problems or symptoms than patients who did not participate in the study. However, we restricted eligibility to enroll the patients who were most likely to benefit from the intervention, a design commonly called targeted or enrichment design. In addition, patients were randomly assigned to the three study groups, and their socio-demographic and clinical characteristics at baseline were not different across the groups.

In addition, this study was conducted at a tertiary cancer hospital in South Korea, and the results may not be generalizable to patients in different settings or in other countries. Yet, chemotherapy regimens for breast cancer are standardized worldwide and chemotherapy-induced skin toxicity is a well-known side effect of breast cancer chemotherapy. However, larger studies from multiple institutions are needed to confirm our findings.

In conclusion, we found that a tailored moisturizer with a high content in pseudo-ceramides improved skin dryness, dullness and dermatological QoL compared to usual care in breast cancer patients undergoing chemotherapy. While additional clinical trials with diverse populations are needed, our trial indicates that a tailored moisturizer may provide beneficial supportive care for breast cancer patients who are likely to experience chemotherapy-induced skin dryness and associated problems.

\section{Methods}

\section{Trial design and participants}

This study was a single-center, randomized controlled trial (registered in Clinical Research Information Service, CRIS: KCT0001372 http://cris.nih.go.kr/cris/en/search/search_result_st01.jsp?seq=6665 and 
date of registration and the date of enrollment of the first participant were 14/01/2015 and 16/02/2015, respectively.) conducted from February 1, 2015 to October 31, 2018 at the Samsung Comprehensive Cancer Center in Seoul, South Korea. We used a 2-phase strategy to recruit study participants with incident (new onset) skin dryness after 1 cycle of chemotherapy. First, we recruited patients 35 to 65 years of age with a postoperative diagnosis of stage I to III breast cancer who were expected to receive doxorubicin plus cyclophosphamide (AC), fluorouracil plus cyclophosphamide and doxorubicin (FAC), or taxane $(\mathrm{T})$ as adjuvant chemotherapy. We excluded patients with severe skin dryness, atopic dermatitis, psoriasis, or infectious skin diseases prior to chemotherapy, as well as patients who were taking steroids, antihistamines, anti-depressants or anticonvulsants. Second, patients who had agreed to participate in the trial prior to chemotherapy were contacted after 1 cycle of chemotherapy and asked about the development of skin dryness to confirm final eligibility.

\section{Randomization and interventions}

Patients were randomized in a 1:1:1 ratio to receive either a tailored moisturizer (1.5\% pseudo-ceramide content), usual care, or a general moisturizer (water-based). The primary comparison was between the tailored moisturizer and usual care. The secondary comparison was between the tailored moisturized and the general moisturizer. Randomization was conducted centrally by an independent statistician using randomly permuted blocks and stratified by menopause status and Herceptin treatment. The allocation information was concealed in sealed envelopes and stored at the investigator's premises until the end of the trial. The sealed code could only be broken at the request of the principal investigator due either to justified serious adverse events or in case of an emergency. Code breaks and their reasons were documented in the case report form.

The tailored moisturizer (cream: Primera, AMOREPACIFIC, Korea) contained $1.5 \%$ of pseudo-ceramides, a lipid that comprises more than $50 \%$ of skin lipids. Patients randomized to the usual care group did not received any products. They were told that they could use any product but were instructed to record the type of products and time of dosing during the study period. The general moisturizer (cream: AMOREPACIFIC, Korea) was a general moisturizing cream with the same color and aroma as the tailored moisturizer. Patients assigned to each moisturizer group were asked to apply the moisturizer twice a day $\left(0.5 \mathrm{ml}\right.$ per application) from the first day of $2^{\text {nd }}$ cycle of chemotherapy until 1 month after completion of chemotherapy. The initial application of moisturizers was performed under the guidance of a clinical researcher. The study products were supplied in bottles containing enough solution for approximately 3 weeks and patients received the products on the first day of each cycle of chemotherapy. Moisturizers could be stored under ambient conditions and no specific storage conditions were required. To assess compliance, patients were asked to return the dispensed bottle. We also asked patients if they had skipped the moisturizer for more than 3 consecutive days.

While it was not possible to blind the assignment of treatment vs. usual care group (control group), assignment to the tailored moisturizer and the general moisturizer groups was blinded. Both moisturizers 
had same texture, smell, and color, and both were supplied in identical $30 \mathrm{~mL}$ plastic bottles. Investigators, outcome assessors, and statisticians were blinded to treatment assignment.

\section{Measurements and outcomes}

The primary outcome was patient-reported severe skin dryness on the face at 1 month after completion of chemotherapy. Skin dryness was assessed by the Patient-Reported Outcomes version of the Common Terminology Criteria for Adverse Events $\left(P R O-C T C A E^{T M}\right){ }^{26,27}$. The PRO-CTCAE question originally included 5 categories that were coded as none (none), moderate (a little or moderate), and severe (severe or very severe). Severe skin dryness was thus defined as severe or very severe dryness using the PRO-CTCAE questionnaire.

The secondary outcomes were two objective skin parameters: cheek surface sebum and water content. Objective skin parameters were measured on the front of the right cheek by trained researchers according to a standard protocol using a Multi-Probe Adapter System (Courage-Khazaka, Germany). Sebum content (approximated to $\mu \mathrm{g} / \mathrm{cm}^{2}$ ) was measured only once per visit, while water content (in arbitrary units, AU) was measured three times per visit and the values were averaged. During measurements, patients were asked to lay face up on a bed keeping a straight body position in all visits at the room maintained temperature between 20 and $22{ }^{\circ} \mathrm{C}$ and humidity between 30 and $40 \%{ }^{28,29}$. All outcomes were measured at baseline (beginning of the $2^{\text {nd }}$ cycle of chemotherapy), 3 weeks after baseline, and 1 month after completion of chemotherapy.

In addition, we assessed patient-reported distress due to skin dryness and dullness as well as skin dryness and dullness using a 10-point visual analog scale with scores ranging from 0 to 10 ( $0=$ not at all, $10=$ very much). Impairment of dermatological quality of life was measured using the Korean version of the Dermatology Life Quality Index (DLQI) ${ }^{30}$. The DLQI consists of 10 simple questions to assess quality of life related to skin problems. Each question had four possible answers scored from 0 to 3 . The answers to all questions were added to obtain an overall score on a scale from 0 to 30 , with higher scores indicating more impairment of dermatological quality of life ${ }^{30}$.

Sociodemographic and behavioral information were collected at baseline using a questionnaire. Clinical information were obtained from electronic medical records. Adverse events were classified and graded according to the Common Terminology Criteria for Adverse Events, version 4.0 [12].

\section{Statistical analysis}

The sample size for the trial was calculated to address the primary hypothesis that the tailored moisturizer was more effective than usual care (primary comparison) in preventing chemotherapyassociated dryness at 1 month after completion of chemotherapy. We then adjusted the sample size to incorporate an additional comparison with the general moisturizer (secondary comparison). Based on prior experience, we expected that $60 \%$ of patients in the usual care group and no more than $30 \%$ of patients in the tailored moisturizer group would report severe dryness on the face 1 month after 
completion of chemotherapy. We adjusted the p-values for two comparisons using the Bonferroni method. To conduct pairwise $\chi^{2}$ tests comparing proportion of severe dryness under Bonferroni adjustment $(2.5 \%$ level of significance; $\mathrm{P}<0.025)$ with $80 \%$ power, the sample size of the trial was 58 patients per arm. Since we expected 15\% losses to follow-up, we increased the sample size to 68 patients per arm, for a total of 204 randomized participants.

All analyses were conducted using the intention-to-treat principle, whereas study patients were assigned to their randomized group irrespective of compliance with the study intervention. Differences in baseline characteristics among 3 groups were compared using $\chi^{2}$ tests for categorical variables and t-tests for continuous variables.

We used mixed effect models for longitudinal data analysis to model changes over time in sebum, and water content. $P$-values $<0.05$ were considered statistically significant except for the primary outcome ( $P$ $<0.025$ ). All analyses were conducted using Stata 15.0 (Stata Corp.; College Station, TX).

\section{Ethical considerations}

This study was conducted in accordance with the Declaration of Helsinki and with local laws and regulations. The study protocol was approved by the Medical Ethical Committee of Samsung Medical Center in Seoul, Korea. Eligible patients were fully informed about the study and volunteered to write informed consent. If during the study a patient no longer wished to participate, for whatever reason, the patient was allowed to withdraw consent at any time. An independent monitoring committee reviewed accumulating safety data throughout the trial.

\section{Protocol changes}

We revised exclusion criteria because of changes in cancer treatment practice caused by changes in insurance reimbursement. Starting on February $2^{\text {nd }}, 2016$, we did not exclude patients who received Herceptin. Starting on August $8^{\text {th }}, 2016$, we did not exclude patients who received taxanes. In addition, to minimize losses to follow-up before patients attended the clinic-based baseline measurement, from November $11^{\text {th }}, 2017$, we changed the time of randomization to the baseline study clinic visit.

\section{Declarations}

\section{Authors' contributions}

DK, JSA, and JC had the original idea for the study. All authors were involved in the study design. DZ undertook initial data management. DK undertook subsequent data management and the primary analysis and wrote the first draft. EG provided advice on data management and analysis. All authors contributed to further drafts and approved the final manuscript.

\section{Ethical Approval}


The study protocol was approved (SMC 2014-11-093) by the Medical Ethical Committee of Samsung Medical Center in Seoul, Korea. This trial were registered in Clinical Research Information Service, CRIS: KCT0001372 (http://cris.nih.go.kr/cris/en/search/search_result_st01.jsp?seq=6665) and date of registration and the date of enrollment of the first participant were 14/01/2015 and 16/02/2015, respectively. Informed consent was obtained from all the participants.

\section{Data Sharing Statement}

Data are available upon reasonable request

\section{Conflicts of interest}

JC received grants from AMOREPACIFIC. HP and EK are the employees of the AMOREPACIFIC. Other all authors no conflict of interest.

\section{Funding}

This work was supported by AMOREPACIFIC and the Korea Breast Cancer Foundation.

\section{Patient consent for publication}

Not required.

\section{References}

1. Stan, D., Loprinzi, C. L. \& Ruddy, K. J. Breast cancer survivorship issues. Hematology/oncology clinics of North America 27, 805-827, http://doi.org/10.1016/j.hoc.2013.05.005 (2013).

2. JJ, G. et al. Factors associated with receipt of breast cancer adjuvant chemotherapy in a diverse population-based sample. J Clin Onco/30, 3058-3064 (2012).

3. Reich, S. \& Trueb, R. M. [Trichoteiromania]. Journal der Deutschen Dermatologischen Gesellschaft = Journal of the German Society of Dermatology : JDDG 1, 22-28 (2003).

4. Kang, D. et al. Quantitative changes in skin composition parameters due to chemotherapy in breast cancer patients: a cohort study. Breast cancer research and treatment 152, 675-682, http://doi.org/10.1007/s10549-015-3502-4 (2015).

5. Katoh, M., Kadota, M. \& Nishimura, Y. A case of docetaxel-induced erythrodysesthesia. The Journal of dermatology 31, 403-406 (2004).

6. Childress, J. \& Lokich, J. Cutaneous hand and foot toxicity associated with cancer chemotherapy. American journal of clinical oncology 26, 435-436, http://doi.org/10.1097/01.coc.0000026486.56886.18 (2003).

7. Karam, A., Metges, J. P., Labat, J. P., Leroy, J. P. \& Guillet, G. Squamous syringometaplasia associated with docetaxel. The British journal of dermatology 146, 524-525 (2002). 
8. Chen, M., Crowson, A. N., Woofter, M., Luca, M. B. \& Magro, C. M. Docetaxel (taxotere) induced subacute cutaneous lupus erythematosus: report of 4 cases. The Journal of rheumatology 31, 818820 (2004).

9. Ascherman, J. A., Knowles, S. L. \& Attkiss, K. Docetaxel (taxotere) extravasation: a report of five cases with treatment recommendations. Annals of plastic surgery 45, 438-441 (2000).

10. Eich, D., Scharffetter-Kochanek, K., Eich, H. T., Tantcheva-Poor, I. \& Krieg, T. Acral erythrodysesthesia syndrome caused by intravenous infusion of docetaxel in breast cancer. American journal of clinical oncology 25, 599-602 (2002).

11. Chu, C. Y., Yang, C. H., Yang, C. Y., Hsiao, G. H. \& Chiu, H. C. Fixed erythrodysaesthesia plaque due to intravenous injection of docetaxel. The British journal of dermatology 142, 808-811 (2000).

12. Viale, P. H. Chemotherapy and cutaneous toxicities: implications for oncology nurses. Seminars in oncology nursing 22, 144-151, http://doi.org/10.1016/j.soncn.2006.04.007 (2006).

13. Bensadoun, R. J. et al. Daily baseline skin care in the prevention, treatment, and supportive care of skin toxicity in oncology patients: recommendations from a multinational expert panel. Cancer management and research 5, 401-408, http://doi.org/10.2147/cmar.S52256 (2013).

14. Ren, Z. et al. Randomized controlled trial of the prophylactic effect of urea-based cream on sorafenib-associated hand-foot skin reactions in patients with advanced hepatocellular carcinoma. $J$ Clin Onco/ 33, 894-900, http://doi.org/10.1200/jco.2013.52.9651 (2015).

15. Lacouture, M. E. et al. Evolving strategies for the management of hand-foot skin reaction associated with the multitargeted kinase inhibitors sorafenib and sunitinib. The oncologist 13, 1001-1011, http://doi.org/10.1634/theoncologist.2008-0131 (2008).

16. Haley, A. C. et al. Skin care management in cancer patients: an evaluation of quality of life and tolerability. Supportive care in cancer: official journal of the Multinational Association of Supportive Care in Cancer 19, 545-554, http://doi.org/10.1007/s00520-010-0851-8 (2011).

17. Luftner, D. et al. Evaluation of supportive and barrier-protective skin care products in the daily prevention and treatment of cutaneous toxicity during systemic chemotherapy. OncoTargets and therapy 11, 5865-5872, http://doi.org/10.2147/ott.S155438 (2018).

18. Imokawa, G. Surfactant-Induced Depletion of Ceramides and Other Intercellular Lipids: Implication for the Mechanism Leading to Dehydration of the Stratum corneum. Exogenous Dermatology 3, 8198, http://doi.org/10.1159/000086158 (2004).

19. Imokawa, G. et al. Pseudo-acylceramide with linoleic acid produces selective recovery of diminished cutaneous barrier function in essential fatty acid-deficient rats and has an inhibitory effect on epidermal hyperplasia. The Journal of clinical investigation 94, 89-96, http://doi.org/10.1172/jci117352 (1994).

20. Cupit-Link, M. C. et al. Biology of premature ageing in survivors of cancer. ESMO open 2, e000250, http://doi.org/10.1136/esmoopen-2017-000250 (2017).

21. Choi, M. J. \& Maibach, H. I. Role of ceramides in barrier function of healthy and diseased skin. American journal of clinical dermatology 6, 215-223, http://doi.org/10.2165/00128071-200506040- 
00002 (2005).

22. Ishida, K., Takahashi, A., Bito, K., Draelos, Z. \& Imokawa, G. Treatment with Synthetic Pseudoceramide Improves Atopic Skin, Switching the Ceramide Profile to a Healthy Skin Phenotype. The Journal of investigative dermatology, http://doi.org/10.1016/j.jid.2020.01.014 (2020).

23. Jonca, N. Ceramides metabolism and impaired epidermal barrier in cutaneous diseases and skin aging: focus on the role of the enzyme PNPLA1 in the synthesis of $\omega$-0-acylceramides and its pathophysiological involvement in some forms of congenital ichthyoses. OCL 26, 17 (2019).

24. Sahle, F. F., Gebre-Mariam, T., Dobner, B., Wohlrab, J. \& Neubert, R. H. Skin diseases associated with the depletion of stratum corneum lipids and stratum corneum lipid substitution therapy. Skin pharmacology and physiology 28, 42-55, http://doi.org/10.1159/000360009 (2015).

25. Takagi, Y., Nakagawa, H., Higuchi, K. \& Imokawa, G. Characterization of surfactant-induced skin damage through barrier recovery induced by pseudoacylceramides. Dermatology (Basel, Switzerland) 211, 128-134, http://doi.org/10.1159/000086442 (2005).

26. Cho, J. et al. Linguistic Validation of the US National Cancer Institute's Patient-Reported Outcomes Version of the Common Terminology Criteria for Adverse Events in Korean. Journal of global oncology 5, 1-10, http://doi.org/10.1200/jgo.18.00193 (2019).

27. Dueck, A. C. et al. Validity and Reliability of the US National Cancer Institute's Patient-Reported Outcomes Version of the Common Terminology Criteria for Adverse Events (PRO-CTCAE). JAMA oncology 1, 1051-1059, http://doi.org/10.1001/jamaoncol.2015.2639 (2015).

28. Pierard, G. E., Pierard-Franchimont, C., Marks, R., Paye, M. \& Rogiers, V. EEMCO guidance for the in vivo assessment of skin greasiness. The EEMCO Group. Skin pharmacology and applied skin physiology 13, 372-389, http://doi.org/29945 (2000).

29. Rogiers, V. EEMCO guidance for the assessment of transepidermal water loss in cosmetic sciences. Skin pharmacology and applied skin physiology 14, 117-128, http://doi.org/56341 (2001).

30. Finlay, A. Y. \& Khan, G. K. Dermatology Life Quality Index (DLQI)-a simple practical measure for routine clinical use. Clinical and experimental dermatology 19, 210-216 (1994).

\section{Tables}


Table 1

Baseline characteristics of study participants $(\mathrm{N}=176)$.

\begin{tabular}{|c|c|c|c|c|}
\hline & $\begin{array}{l}\begin{array}{l}\text { Tailored } \\
\text { moisturizer }\end{array} \\
(N=59)\end{array}$ & $\begin{array}{l}\begin{array}{l}\text { Usual } \\
\text { care }\end{array} \\
(N=61)\end{array}$ & $\begin{array}{l}\text { General } \\
\text { moisturizer } \\
(N=56)\end{array}$ & $P$-value \\
\hline Age at diagnosis (years) & $48.0(8.53)$ & $48.2(7.1)$ & $48.3(8.2)$ & 0.98 \\
\hline Marital status (married) & $51(86.4)$ & $57(93.4)$ & $46(82.1)$ & 0.17 \\
\hline $\begin{array}{l}\text { Education status (> high } \\
\text { school) }\end{array}$ & $34(57.6)$ & $33(54.1)$ & $31(55.4)$ & 0.93 \\
\hline Employ status & & & & 0.25 \\
\hline Housewife or retired & $39(66.1)$ & $32(52.5)$ & $30(53.6)$ & \\
\hline Employee or self-business & $20(33.9)$ & $29(47.5)$ & $26(46.4)$ & \\
\hline Monthly family income & & & & 0.30 \\
\hline$<\$ 5,000$ & $28(47.5)$ & $24(39.3)$ & $30(53.6)$ & \\
\hline$\geq \$ 5,000$ & $31(52.2)$ & $37(60.7)$ & $26(46.4)$ & \\
\hline Drinking status & & & & 0.33 \\
\hline Never drinker & $38(64.4)$ & $35(57.4)$ & $26(46.4)$ & \\
\hline Past drinker & $20(33.9)$ & $23(37.7)$ & $28(50.0)$ & \\
\hline Current drinker & $1(1.7)$ & $3(4.9)$ & $2(3.6)$ & \\
\hline Smoking status & & & & 0.06 \\
\hline Never smoker & $56(94.9)$ & $60(98.4)$ & $49(87.5)$ & \\
\hline Past smoker & $1(1.7)$ & $1(1.6)$ & $6(10.7)$ & \\
\hline Current smoker & $2(3.4)$ & 0 & $1(1.8)$ & \\
\hline Disease stage at diagnosis & & & & 0.16 \\
\hline Stage 1 & $26(44.1)$ & $19(31.2)$ & $18(32.1)$ & \\
\hline Stage 2 & $22(37.3)$ & $32(52.5)$ & $33(58.9)$ & \\
\hline Stage 3 & 11 (18.6) & 10 (16.4) & $5(8.9)$ & \\
\hline Regimen & & & & 0.67 \\
\hline
\end{tabular}

Values in the Table are mean (SD) or number (\%).

AC, doxorubicin, cyclophosphamide; FAC, fluorouracil, doxorubicin, cyclophosphamide; T, taxotere; TAC, taxotere, adriamycin and cyclophosphamide; TC, taxotere and cyclophosphamide. 


\begin{tabular}{|lllll|}
\hline & $\begin{array}{l}\text { Tailored } \\
\text { moisturizer } \\
(\mathbf{N = 5 9 )}\end{array}$ & $\begin{array}{l}\text { Usual } \\
\text { care } \\
(\mathbf{N = 6 1 )}\end{array}$ & $\begin{array}{l}\text { General } \\
\text { moisturizer } \\
(\mathbf{N = 5 6 )}\end{array}$ & P-value \\
\hline AC & $27(45.8)$ & $35(57.4)$ & $25(44.6)$ & \\
\hline TAC & $2(3.4)$ & $1(1.6)$ & $4(7.1)$ & \\
\hline TC & $7(11.9)$ & $7(11.5)$ & $8(14.3)$ & 0.28 \\
\hline AC + T & $19(32.2)$ & $17(27.9)$ & $16(28.6)$ & 0.89 \\
\hline Others & $4(6.8)$ & $1(1.6)$ & $3(5.4)$ & 0.74 \\
\hline Hormone therapy & $42(71.2)$ & $49(80.3)$ & $38(67.9)$ & \\
\hline Targeted therapy & $7(11.9)$ & $7(11.5)$ & $8(14.3)$ & \\
\hline Menopause & $23(39.0)$ & $21(34.4)$ & $18(32.1)$ & \\
\hline Values in the Table are mean (SD) or number (\%). & & & \\
\hline AC, doxorubicin, cyclophosphamide; FAC, fluorouracil, doxorubicin, cyclophosphamide; T, taxotere; \\
\hline TAC, taxotere, adriamycin and cyclophosphamide; TC, taxotere and cyclophosphamide.
\end{tabular}


Table 2

Average difference in sebum and water contents from baseline by treatment group.

$\begin{array}{lll}\begin{array}{l}\text { Tailored } \\ \text { moisturizer }\end{array} & \text { Usual care } & \begin{array}{l}\text { General } \\ \text { moisturizer }\end{array}\end{array}$

Sebum content in the cheek $\left(\mu \mathrm{g} / \mathrm{cm}^{2}\right)$

\begin{tabular}{|c|c|c|c|}
\hline Baseline, mean (SD) & $3.29(3.64)$ & $5.48(9.95)$ & $6.23(10.21)$ \\
\hline 3 weeks, mean (SD) & $4.73(7.29)$ & $2.44(3.48)$ & $3.96(8.02)$ \\
\hline Change from baseline $(95 \% \mathrm{Cl})$ & $\begin{array}{l}1.33(-0.89 \\
3.55)\end{array}$ & $\begin{array}{l}-3.05(-5.40 \\
-0.70)\end{array}$ & $\begin{array}{l}-2.11(-4.51 \\
0.29)\end{array}$ \\
\hline Difference between groups $(95 \% \mathrm{Cl})$ & Reference & $\begin{array}{l}-4.38(-7.61 \\
-1.14)\end{array}$ & $\begin{array}{l}-3.44(-6.72 \\
-0.17)\end{array}$ \\
\hline$P$ value compared to tailored moisturizer & & $<0.01$ & 0.04 \\
\hline $\begin{array}{l}1 \text { month after completion of chemotherapy, } \\
\text { mean (SD) }\end{array}$ & $4.38(5.76)$ & $2.89(4.36)$ & $5.22(11.24)$ \\
\hline Change from baseline $(95 \% \mathrm{Cl})$ & $\begin{array}{l}0.77(-1.53 \\
3.08)\end{array}$ & $\begin{array}{l}-2.64(-5.03 \\
-0.25)\end{array}$ & $\begin{array}{l}-0.96(-3.36 \\
1.45)\end{array}$ \\
\hline Difference between groups (95\% Cl) & Reference & $\begin{array}{l}-3.41(-6.73 \\
-0.09)\end{array}$ & $\begin{array}{l}-1.73(-5.06, \\
1.60)\end{array}$ \\
\hline
\end{tabular}

$P$ value compared to tailored moisturizer

$0.04 \quad 0.31$

Water content in the cheek (AU)

\begin{tabular}{|c|c|c|c|}
\hline Baseline, mean (SD) & $86.27(16.68)$ & $82.41(16.82)$ & $86.35(35.69)$ \\
\hline 3 weeks after intervention, mean (SD) & $86.15(20.37)$ & $95.33(17.53)$ & $79.93(16.71)$ \\
\hline Change from baseline $(95 \% \mathrm{Cl})$ & $\begin{array}{l}-0.20(-6.57 \\
6.18)\end{array}$ & $\begin{array}{l}-0.94(-7.76 \\
5.88)\end{array}$ & $\begin{array}{l}-2.32(-9.23 \\
4.69)\end{array}$ \\
\hline Difference between groups $(95 \% \mathrm{Cl})$ & Reference & $\begin{array}{l}-0.74(-10.07 \\
8.59)\end{array}$ & $\begin{array}{l}-2.13(-11.52, \\
7.27)\end{array}$ \\
\hline$P$ value compared to tailored moisturizer & & 0.88 & 0.66 \\
\hline $\begin{array}{l}1 \text { month after completion of chemotherapy, } \\
\text { mean (SD) }\end{array}$ & $88.44(16.84)$ & $86.94(14.38)$ & $82.37(18.82)$ \\
\hline Change from baseline $(95 \% \mathrm{Cl})$ & $\begin{array}{l}2.15(-4.20, \\
8.50)\end{array}$ & $\begin{array}{l}0.53(-6.15, \\
7.21)\end{array}$ & $\begin{array}{l}-0.10(-6.80, \\
6.60)\end{array}$ \\
\hline Difference between groups $(95 \% \mathrm{Cl})$ & Reference & $\begin{array}{l}-1.61(-10.83 \\
7.60)\end{array}$ & $\begin{array}{l}-2.25(-11.48 \\
6.98)\end{array}$ \\
\hline$P$ value compared to tailored moisturizer & & 0.73 & 0.63 \\
\hline
\end{tabular}


Table 3

Patient reported skin dryness, dullness, associated stress, and Dermatology Life Quality Index at 1 month after completion of chemotherapy by treatment group.

\begin{tabular}{|c|c|c|c|c|c|}
\hline & $\begin{array}{l}\text { Tailored } \\
\text { moisturizer } \\
(N=59)\end{array}$ & $\begin{array}{l}\begin{array}{l}\text { Usual } \\
\text { care }\end{array} \\
(N= \\
61)\end{array}$ & $\begin{array}{l}\text { General } \\
\text { moisturizer } \\
(N=56)\end{array}$ & $\begin{array}{l}\text { Tailored } \\
\text { moisturizer vs. } \\
\text { usual care } \\
P \text {-value }\end{array}$ & $\begin{array}{l}\text { Tailored } \\
\text { moisturizer vs. } \\
\text { general } \\
\text { moisturizer } \\
P \text {-value }\end{array}$ \\
\hline Dryness (0-10) & $3.8(2.6)$ & $\begin{array}{l}5.4 \\
(2.2)\end{array}$ & $4.4(2.5)$ & $<0.01$ & 0.18 \\
\hline $\begin{array}{l}\text { Stress due to } \\
\text { dryness }(0-10)\end{array}$ & $1.5(2.4$ & $\begin{array}{l}2.8 \\
(3.1)\end{array}$ & $1.8(2.6)$ & 0.01 & 0.58 \\
\hline Dullness $(0-10)$ & $3.1(3.0)$ & $\begin{array}{l}4.6 \\
(3.0)\end{array}$ & $4.4(3.1)$ & 0.01 & 0.02 \\
\hline $\begin{array}{l}\text { Stress due to } \\
\text { dullness }(0-10)\end{array}$ & $1.7(2.9)$ & $\begin{array}{l}3.5 \\
(3.3)\end{array}$ & $2.8(3.0)$ & $<0.01$ & 0.07 \\
\hline DLQI (0-30) & $2.0(2.8)$ & $\begin{array}{l}4.8 \\
(4.7)\end{array}$ & $3.9(4.2)$ & $<0.001$ & 0.02 \\
\hline \multicolumn{6}{|c|}{ Values were mean (SD). } \\
\hline
\end{tabular}

Figures 


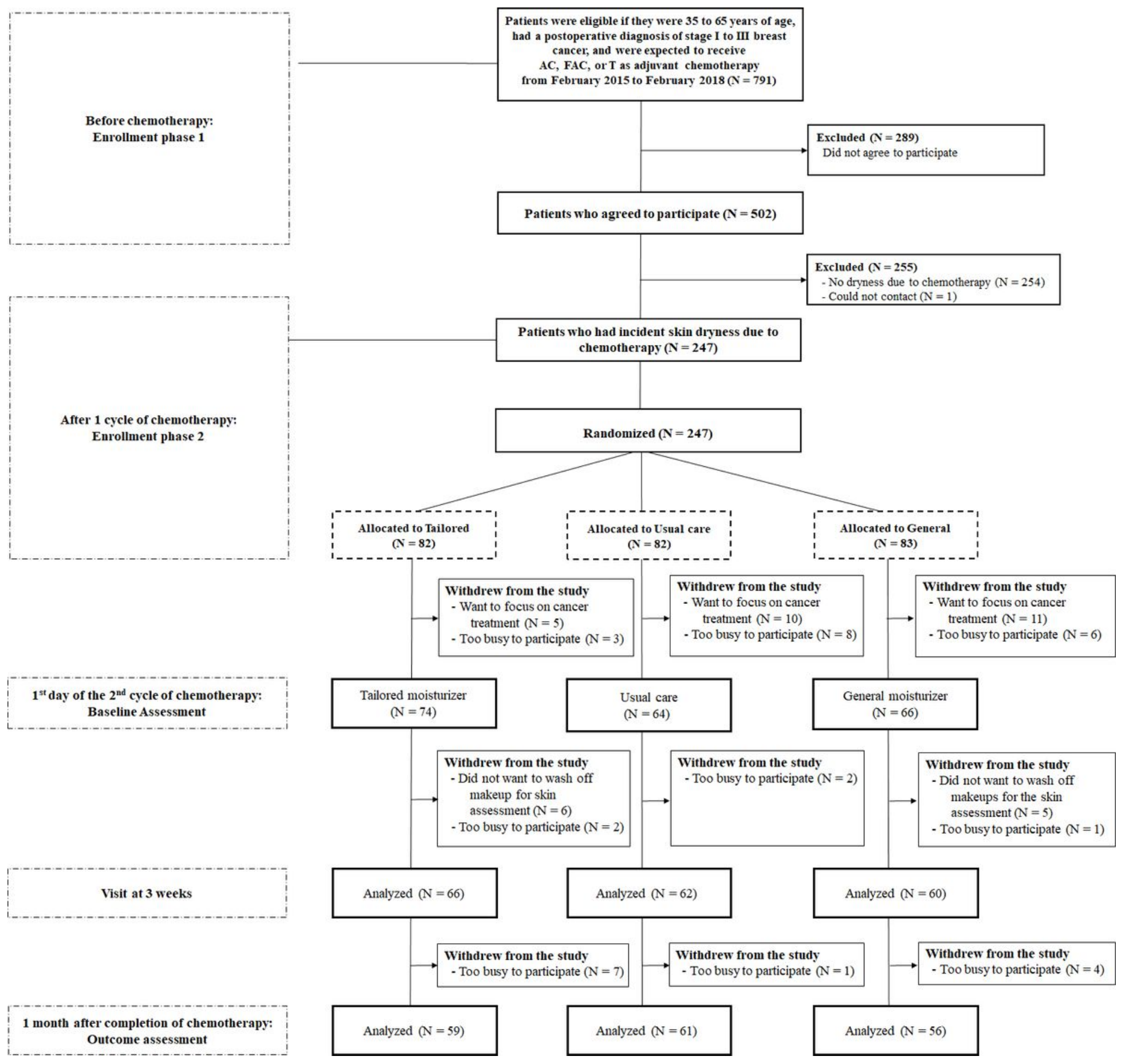

Figure 1

CONSORT flow diagram of study participants. 

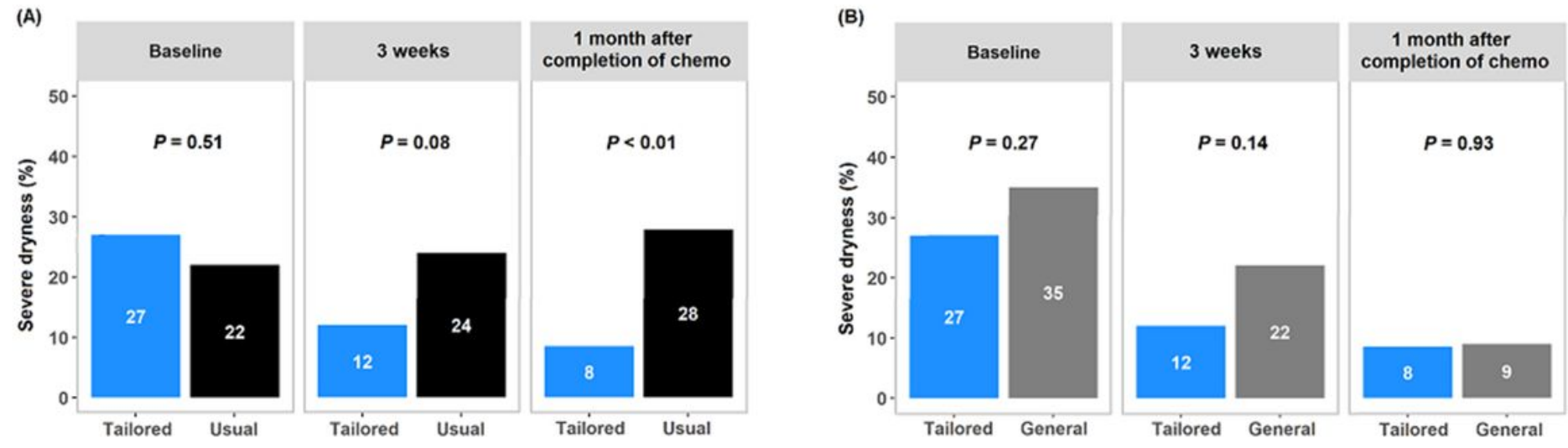

Figure 2

Proportion of patients with severe skin dryness in the tailored moisturizer group compared to the usual care (A) and general moisturizer (B) groups.

(A)

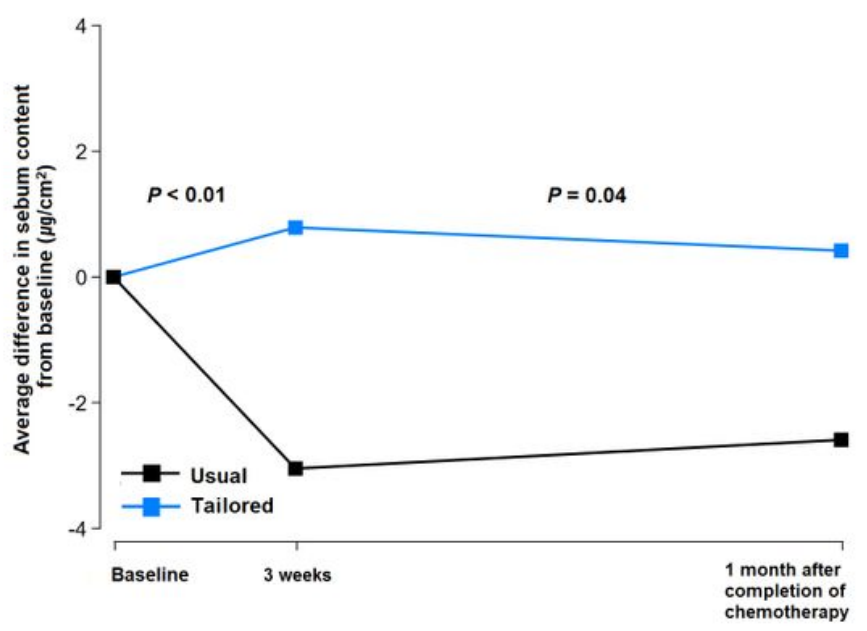

(B)

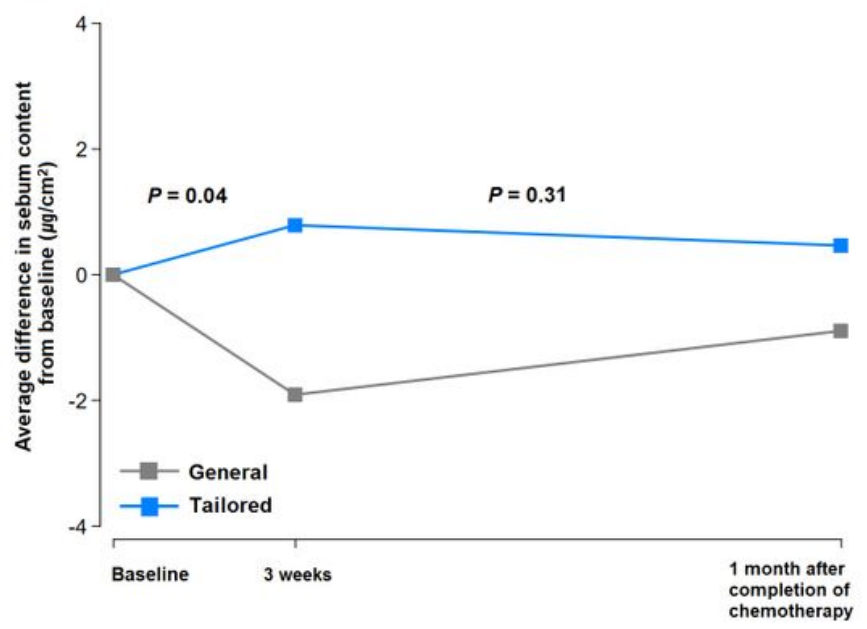

\section{Figure 3}

Average difference in sebum content in the cheek from baseline in the tailored moisturizer group compared to the usual care (A) and the general moisturizer (B) groups.

\section{Supplementary Files}

This is a list of supplementary files associated with this preprint. Click to download.

- DERMARCTsupplement.docx 